\title{
Plasma biomarkers for distinguishing etiologic subtypes of thoracic aortic aneurysm disease
}

\author{
John S. Ikonomidis, MD, PhD, ${ }^{\mathrm{a}}$ Charlotte R. Ivey, BS, ${ }^{\mathrm{a}}$ Jason B. Wheeler, MD, ${ }^{\mathrm{a}}$ Adam W. Akerman, BS, ${ }^{\mathrm{a}}$ \\ Allison Rice, BS, ${ }^{a}$ Risha K. Patel, BS, ${ }^{a}$ Robert E. Stroud, MS, ${ }^{a}$ Asad A. Shah, MD, ${ }^{b}$ Chad G. Hughes, MD, \\ Giovanni Ferrari, $\mathrm{PhD},{ }^{\mathrm{c}}$ Rupak Mukherjee, $\mathrm{PhD},{ }^{\mathrm{a}}$ and Jeffrey A. Jones, $\mathrm{PhD}{ }^{\mathrm{a}, \mathrm{d}}$
}

Background: Thoracic aortic aneurysms (TAAs) develop through an asymptomatic process resulting in gross
dilation that progresses to rupture if left undetected and untreated. If detected, patients with TAA are followed
over time until the risk of rupture outweighs the risk of surgical repair. Current methodologies for tracking TAA
size are limited to expensive computed tomography or magnetic resonance imaging because no acceptable popula-
tion screening tools are currently available. Previous studies from this laboratory and others have identified differ-
ential protein profiles for the matrix metalloproteinases (MMPs) and their endogenous tissue inhibitors (TIMPs), in
ascending TAA tissue from patients with bicuspid aortic valves (BAVs), versus patients with idiopathic degenerative
disease and a tricuspid aortic valve (TAV). In addition, altered microRNA (miR) expression levels have also been
reported in TAAs compared with normal aortic tissue. The objective of our study was to identify circulating factors
within plasma that could serve as potential biomarkers for distinguishing etiologic subtypes of aneurysm disease.

Methods: Ascending TAA tissue and plasma specimens were obtained from patients with BAV $(\mathrm{n}=21)$ and TAV $(\mathrm{n}=21)$ at the time of surgical resection. The protein abundance of key MMPs $(1,2,3,8$, and 9), TIMPs $(1,2,3$, and 4$)$, and miRs $(1,21,29 a, 133 a, 143$, and 145) was examined using a multianalyte protein profiling system or by quantitative polymerase chain reaction, respectively. Results were compared with normal aortic tissue and plasma obtained from patients without aortic disease $(\mathrm{n}=10)$.

Results: Significant $(P<.05)$ differences in standardized miR-1 and miR-21 abundance between BAV and TAV aortic tissue samples and different tissue and plasma profiles of analyte differences from normal aorta where observed between the BAV and TAV groups. Linear regression analysis revealed significant linear relationships in plasma and tissue measurements only for MMP-8 and TIMP-1, TIMP-3, and TIMP-4 $(P<.05)$. Receiver operator curve analysis revealed specific cassettes of analytes predictive of TAA disease. Relative to normal aorta, BAV proteolytic balance was significantly increased for MMP-1, MMP-2, and MMP-7, and for decreased MMP-8 and MMP-9. In contrast, TAV proteolytic balance relative to normal aorta was significantly increased only for MMP-1 and decreased for MMP-8 and MMP-9.

Conclusions: Taken together, these unique data demonstrate differential plasma profiles of MMPs, TIMPs, and miRs in ascending TAA specimens from patients with BAV and TAV. These results suggest that circulating biomarkers may form the foundation for a broader platform of biomarkers capable of detecting the presence of TAA using a simple blood test and may also be useful in personalized strategies to distinguish between etiologic subtypes of TAAs in patients with aneurysm disease. (J Thorac Cardiovasc Surg 2013;145:1326-33)

From the Division of Cardiothoracic Surgery, ${ }^{a}$ Department of Surgery, Medical University of South Carolina, Charleston, SC; Division of Cardiothoracic Surgery, ${ }^{\mathrm{b}}$ Department of Surgery, Duke University School of Medicine, Durham, NC; Department of Surgery, ${ }^{c}$ Perelman School of Medicine, University of Pennsylvania School of Medicine, Philadelphia, Pa; and Research Service, ${ }^{\mathrm{d}}$ Ralph H. Johnson Veterans Affairs Medical Center, Charleston, SC.

This study was supported by the following grants: National Institutes of Health/ National Institute on Aging No. AG036954, National Institutes of Health/National Heart, Lung, and Blood Institute No. HL102121, and Veterans Affairs Merit Award No. 1 I01 BX000904-01.

Disclosures: Authors have nothing to disclose with regard to commercial support.

Read at the 38th Annual Meeting of The Western Thoracic Surgical Association, Maui, Hawaii, June 27-30, 2012.

Received for publication June 25, 2012; revisions received Nov 4, 2012; accepted for publication Dec 10, 2012; available ahead of print Jan 14, 2013.

Address for reprints: John S. Ikonomidis MD, PhD, FRCS(C), FACS, FAHA, FACC, Medical University of South Carolina, 25 Courtenay Dr, Suite 7030, Charleston, SC 29425 (E-mail: ikonomij@musc.edu).

$0022-5223 / \$ 36.00$

Copyright (c) 2013 by The American Association for Thoracic Surgery

http://dx.doi.org/10.1016/j.jtcvs.2012.12.027
Thoracic aortic aneurysm (TAA) is an insidious and potentially devastating disease process. Despite advancements in our understanding of the pathobiology of TAAs, these findings have yet to be translated into significant clinical applications for screening, diagnosis, tracking, and treatment of TAAs.

From a biologic standpoint, numerous studies have confirmed that specific proteinases such as the matrix metalloproteinases (MMPs) and their endogenous inhibitors (TIMPs) are implicated in the pathogenesis of ascending TAAs. ${ }^{1-4}$ In addition, specific and different cassettes of MMPs and TIMPs have been demonstrated in ascending TAAs with different etiologies, such as those associated with congenitally bicuspid aortic valves (BAVs) or tricuspid aortic valves (TAVs). ${ }^{1-4}$ Similar findings have 

Abbreviations and Acronyms
AUC = area under the curve
$\mathrm{BAV}=$ bicuspid arterial valve
$\mathrm{Ct}=$ cycle threshold
$\mathrm{miR}=$ microRNA
MMP $=$ matrix metalloproteinase
MSA $=$ multiplex suspension array
$\mathrm{TAA}=$ thoracic aortic aneurysm
TIMP $=$ tissue inhibitors of metalloproteinase

been demonstrated with microRNAs (miRs): different types are seen to be expressed within these aneurysms. ${ }^{5}$ Many of these agents can be reliably measured in plasma, providing a potentially novel strategy to identify and follow the progression of TAAs. Accordingly, we sought to identify circulating plasma factors associated with TAA that could distinguish and predict the presence and etiologic subtype of aneurysm disease.

\section{METHODS}

\section{Patient Demographics}

Matched tissue and plasma specimens from 42 patients with ascending aortic aneurysms ( 21 patients with BAV and 21 patients with TAV) were taken from the widest region of the ascending aorta at the time of surgical resection or aortic valve replacement. No patients had aortic dissection, inflammatory aortic disease, or known connective tissue disorder. Normal aortic specimens were similarly harvested from the ascending aorta of heart transplant donors or recipients $(\mathrm{n}=10)$. Mean \pm standard deviation ages were $58 \pm 6$ years in the normal group, $59 \pm 2$ years in the BAV group, and $70 \pm 2$ years in the TAV group (TAV $P<.05$ from BAV and normal). Seventy percent of normal group patients, $71 \%$ of BAV group patients, and $52 \%$ of TAV group patients were men. Aortic diameters were $3.8 \pm 0.2 \mathrm{~cm}$ in the normal group, $5.2 \pm 0.2 \mathrm{~cm}$ in the BAV group, and $5.7 \pm 0.2 \mathrm{~cm}$ in the TAV group (TAV and BAV $P<.05$ from normal). Normal aortic tissue and plasma specimens were snap frozen and stored at $-80^{\circ} \mathrm{C}$ until analyzed. This study was approved by the institutional review boards of the Medical University of South Carolina, Duke University, and the University of Pennsylvania. Informed consent was obtained from all patients.

\section{miR Isolation}

Tissue samples. For each tissue sample, $5 \mathrm{mg}$ frozen tissue was weighed and homogenized using a bead-mill homogenizer (Qiagen, Valencia, Calif). Total RNA was extracted from tissue homogenates (mirVana PARIS miRNA Isolation kit; Applied Biosystems/Ambion, Austin, Tex) and analyzed for RNA quality and quantity using an Experion Automated Electrophoresis System (RNA StdSens Analysis Kit; Bio-Rad Laboratories, Hercules, Calif). Ten nanograms total RNA was reversed transcribed (TaqMan MicroRNA Reverse Transcription Kit; Applied Biosystems/Ambion, Austin, Tex) for each miR of interest, and quantitative polymerase chain reaction (PCR) was performed. Each tissue sample was analyzed for the following miRs: miR-1, miR-21, miR-29a, miR-133a, miR-143, and miR-145. These were selected because previous of previous findings by our group, ${ }^{5}$ their reported involvement in the regulation of specific protein targets within the cardiovascular system, including several MMPs and extracellular matrix components (eg, collagens, elastin, and microfibrillar proteins),${ }^{6}$ and because of a previous report of involvement (miR-143/miR-145) in ascending TAA formation. ${ }^{7}$
Plasma samples. RNA was isolated from $50 \mu \mathrm{L}$ plasma (mirVana PARIS Protein and RNA isolation System for Small RNAs; Ambion, Austin, Tex) following the manufacturer's instructions. The isolated RNA ( $40 \mu \mathrm{L}$ ) was then incubated for 1 hour at room temperature with 1.3 units Heparinase-I (Ibex Pharmaceuticals Inc, Montreal, Quebec, Canada) in a buffer containing $20 \mathrm{mM}$ tris(hydroxymethyl)aminomethane ( $\mathrm{pH}$ 7.5), $50 \mathrm{mM}$ sodium chloride, $4 \mathrm{mM}$ calcium chloride, and $0.01 \%$ bovine serum albumin. Five microliters treated RNA was reverse transcribed for each miR of interest and quantitative PCR was performed. Each plasma sample was analyzed for the following miRs: miR-1, miR-21, miR-29a, miR-133a, miR-143, and miR-145.

\section{Quantitative PCR}

For both tissue and plasma samples, the reverse transcription product was amplified with gene-specific TaqMan primer/probe sets using the TaqMan Universal PCR Master Mix with no AmperErase UNG (Catalog No. 4324020; Applied Biosystems, Carlsbad, Calif) in a CFX96 RealTime PCR Detection System (Bio-Rad, Hercules, Calif). The thermal cycling protocol was conducted as follows: 10 minutes at $95^{\circ} \mathrm{C}$, followed by 40 cycles of $95^{\circ} \mathrm{C}$ for 15 seconds, and $60^{\circ} \mathrm{C}$ for 1 minute. Negative PCR controls were run to verify the absence of genomic DNA contamination (no reverse transcription control). Fluorescence was recorded at regular intervals following the $60^{\circ} \mathrm{C}$ annealing/extension segment of the PCR reaction and real-time data showing relative fluorescence versus cycle number was analyzed. Because of the paucity of good internal PCR controls for plasma specimens, miR expression in both tissue and plasma (for consistency of measurement) was determined from a change in cycle threshold $(\Delta \mathrm{Ct})$ value (expression $=2^{(-\Delta \mathrm{Ct})}$ ) where $\Delta \mathrm{Ct}$ was derived for each individual specimen, and calculated by subtracting the mean $\mathrm{Ct}$ value for all targets measured from the individual $\mathrm{Ct}$ value of a given PCR target, as previously described. ${ }^{8,9}$ Results were then reported as a mean \pm standard error for each miR measured in either tissue or plasma.

\section{MMP/TIMP Multiplex Suspension Array (MSA)}

Utilizing an MSA approach, the tissue specimens were thawed and transferred to a cold buffer (volume 1:6 w/v) containing $10 \mathrm{mM}$ cacodylic acid ( $\mathrm{pH} 5.0$ ), $0.15 \mathrm{M}$ sodium chloride, $10 \mathrm{mM}$ zinc chloride, $1.5 \mathrm{mM}$ sodium azide, and $0.01 \%$ Triton X-100 (v/v; Cat T8787, Sigma-Aldrich, St Louis, Mo), and homogenized using a bead-mill homogenizer (Qiagen, Valencia, Calif). The homogenates were then centrifuged $(800 \times \mathrm{g}, 10$ minutes, $4^{\circ} \mathrm{C}$ ), and $20 \mu \mathrm{g}$ protein was analyzed using an MSA approach. MMPs 1, 2, 3, 7, 8, 9, 12, and 13 and TIMPs 1, 2, 3, and 4 were examined as previously described. ${ }^{10}$ The plasma specimens were analyzed in a similar fashion following dilution (1:100 for MMP-2 and MMP-9; 1:10 for MMP1, MMP-3, MMP-7, MMP-8, MMP-12, and MMP-13; and 1:20 for the TIMPs), as previously described. ${ }^{11}$ In both cases, samples were incubated on a microplate shaker (room temperature, 2 hours), filtered, and washed 3 times with $100 \mu \mathrm{L}$ wash buffer. Diluted goat antihuman polyclonal biotinylated antibodies $(50 \mu \mathrm{L}$, analyte specific [included with antibodyconjugated bead kits]; R\&D Systems, Inc, Minneapolis, Minn) were then added to each well and the specimens were again incubated on a microplate shaker (room temperature, 1 hour). The beads were filtered and washed as before, and streptavidin-phycoerythrin (50 $\mu \mathrm{L}, \mathrm{R} \& \mathrm{D}$ Systems, Inc) was added to each well for 30 minutes at room temperature. After a final filtration and wash, the beads were analyzed using the Bio-Plex System; fluorescence was measured and then compared with standard curves for each analyte also run on the same plate. Protein quantities were calculated using Bio-Plex Manager Software (version 4.1) and expressed as absolute concentration in picograms per milliliter.

\section{Data Analysis}

Expression levels of miRs and protein abundance of the MMPs/TIMPs were analyzed in 2 ways. First, all quantitative PCR and MSA results were 
$\underline{\text { Tissue }}$
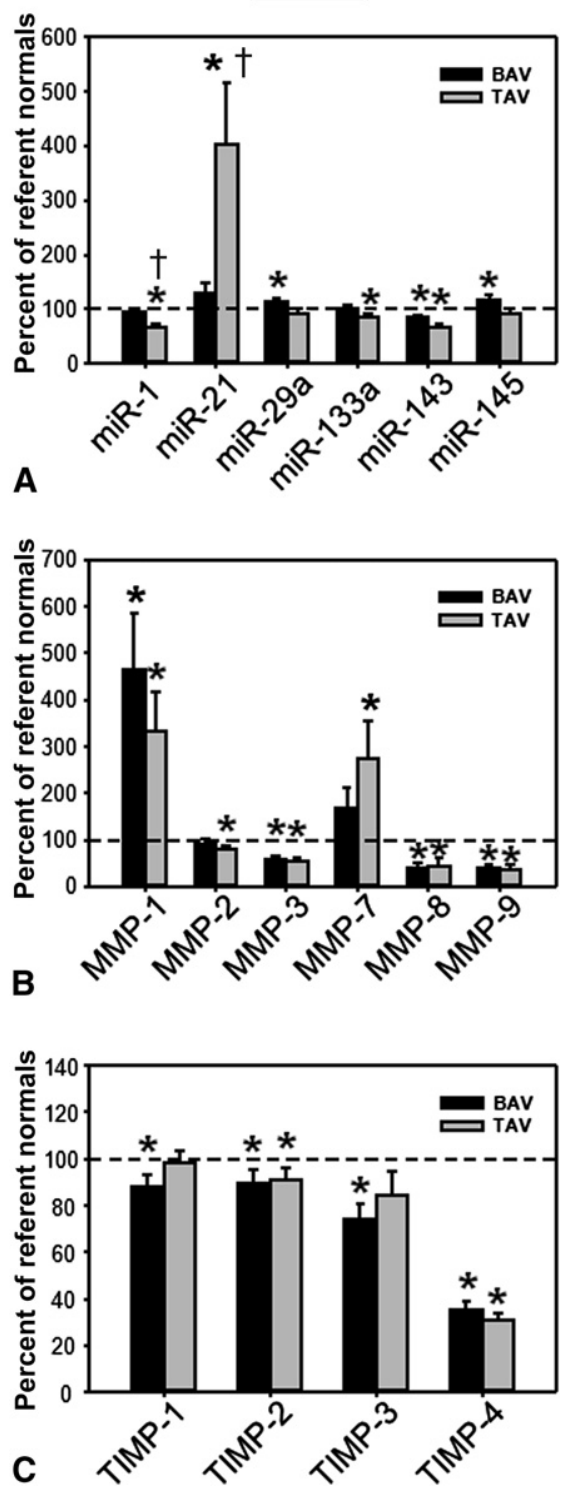

Plasma
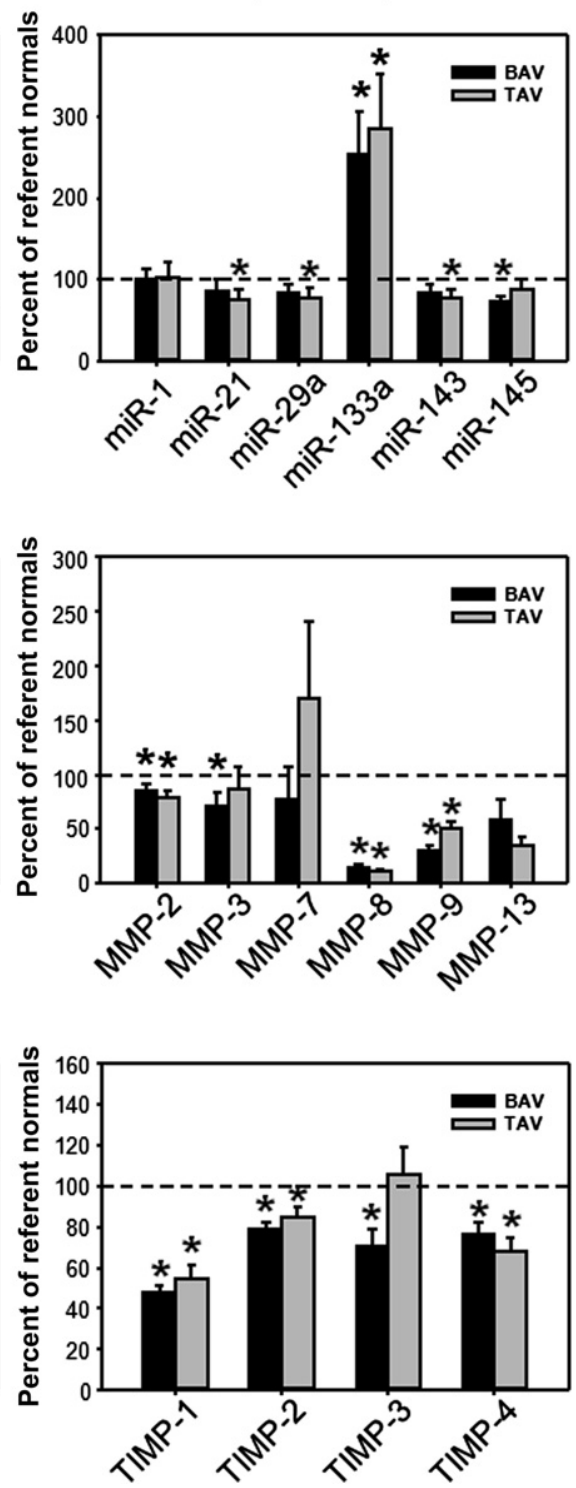

FIGURE 1. Aortic tissue and plasma analysis for microRNAs (miRs), matrix metalloproteinases (MMPs) and tissue inhibitors of matalloproteinases $(T I M P s)$, comparing ascending thoracic aortic aneurysms (TAAs) associated with bicuspid aortic valve (BAV) or tricuspid aortic valve (TAV) from normal aortic samples (dashed line). Significant differences were observed between the BAV and TAV groups, and between the aneurysm groups and normal aorta group. $* P<.05$ from normal aorta. $\dagger P<.05$ from BAV.

subjected to a Shapiro-Wilk test for normality. For the unequally distributed analytes, the absolute values were $\log$ transformed. Then all values were subjected to a 1-way analysis of variance (prcomp module of Stata version 8.2; Stata Corp, College Station, Tex) with Tukey's wholly significant difference post hoc analysis for separation of means to determine differences between the referent controls, BAV, and TAV groups. Second, the percent change of miR and MMP/TIMP levels in the BAV and TAV groups were computed and compared with the referent controls using a 1-sample mean comparison test with the hypothesized mean set at $100 \%$. Analysis of variance with Tukey's wholly significant difference post hoc analysis (prcomp module) was used to determined differences between BAV and TAV groups. Linear regression analysis was performed to identify significant relationships between tissue and plasma levels of each analyte. In addition, plasma biomarkers were assessed for univariate association with the presence of aortic aneurysm using logistic regression models. Receiver operating characteristic curves was then generated to compute an area under the curve (AUC) for each individual biomarker. Biomarkers with $P$ values $<.25$ were considered for inclusion in a multiple logistic regression model. Using forward stepwise variable selection, biomarkers were added to the model with the variable most strongly associated with outcome (presence of a TAA) being added to the model until no more variables met the entry criterion of $\alpha \leq 0.20$. The $\alpha$ level was set at 0.20 to ensure that even marginally predictive biomarkers were captured. Logistic regression analysis was performed to determine the coefficients and intercepts for biomarkers that were found to be significant predictors of aneurysm development in multivariable analysis. Discrimination and classification of the fitted multivariable models were assessed by using the generated equation and computing the corresponding sensitivity, specificity, positive 
TABLE 1. Absolute values for microRNA (miR) expression and protein abundance of matrix metalloproteinases (MMPs) and tissue inhibitors of metalloproteinase (TIMPs) in aortic tissue from healthy patients and patients with thoracic aortic aneurism (TAA) with bicuspid aortic valve (BAV) or tricuspid aortic valve (TAV)

\begin{tabular}{|c|c|c|c|c|}
\hline & Tissue analyte & Control & BAV & TAV \\
\hline \multicolumn{5}{|l|}{$\operatorname{miR} \mathrm{A}\left(2^{-\Delta \mathrm{Ct}}\right)$} \\
\hline & $\operatorname{miR}-1\left(1 \times 10^{-3}\right)$ & $57.8 \pm 2.4$ & $54.3 \pm 3.3$ & $39.4 \pm 3.3^{*}, \dagger$ \\
\hline & $\operatorname{miR}-21\left(1 \times 10^{-2}\right)$ & $169.7 \pm 21.4$ & $217.7 \pm 32.3$ & $683.0 \pm 191.0^{*}, \dagger$ \\
\hline & $\operatorname{miR}-29 \mathrm{a}\left(1 \times 10^{-2}\right)$ & $242.4 \pm 33.3$ & $274.7 \pm 17.2$ & $220.2 \pm 25.8$ \\
\hline & miR-133a $\left(1 \times 10^{-3}\right)$ & $80.3 \pm 5.2$ & $81.7 \pm 5.8$ & $69.2 \pm 5.1$ \\
\hline & $\operatorname{miR}-143\left(1 \times 10^{-2}\right)$ & $830.9 \pm 37.9$ & $703.7 \pm 45.9$ & $560.6 \pm 49.1^{*}$ \\
\hline & $\operatorname{miR}-145\left(1 \times 10^{-2}\right)$ & $771.0 \pm 37.9$ & $910.8 \pm 72.1$ & $715.0 \pm 75.6$ \\
\hline \multicolumn{5}{|l|}{ MMPs (pg/mL) } \\
\hline & MMP-1 & $3.4 \pm 1.0$ & $15.7 \pm 4.0$ & $11.3 \pm 2.8$ \\
\hline & MMP-2 $\left(1 \times 10^{2}\right)$ & $71.6 \pm 13.0$ & $64.1 \pm 7.7$ & $56.9 \pm 5.4$ \\
\hline & MMP-3 & $292.9 \pm 45.5$ & $165.2 \pm 22.4$ & $160.6 \pm 20.2$ \\
\hline & MMP-7 $\left(1 \times 10^{1}\right)$ & $8.8 \pm 2.9$ & $14.6 \pm 3.8$ & $24.0 \pm 7.2$ \\
\hline & MMP-8 $\left(1 \times 10^{1}\right)$ & $294.1 \pm 128.8$ & $121.7 \pm 29.3$ & $128.5 \pm 49.8$ \\
\hline & MMP-9 $\left(1 \times 10^{2}\right)$ & $74.9 \pm 49.6$ & $28.3 \pm 7.1$ & $26.0 \pm 7.9$ \\
\hline & MMP-12 & ND & ND & ND \\
\hline & MMP-13 & ND & ND & ND \\
\hline \multicolumn{5}{|l|}{ TIMPs (pg/mL) } \\
\hline & TIMP-1 $\left(1 \times 10^{2}\right)$ & $113.6 \pm 13.7$ & $100.2 \pm 5.9$ & $111.3 \pm 6.1$ \\
\hline & TIMP-2 $\left(1 \times 10^{2}\right)$ & $127.9 \pm 17.1$ & $114.2 \pm 7.5$ & $116.3 \pm 6.5$ \\
\hline & TIMP-3 $\left(1 \times 10^{2}\right)$ & $22.2 \pm 3.0$ & $16.5 \pm 1.5$ & $18.8 \pm 2.2$ \\
\hline & TIMP-4 & $141.9 \pm 33.7$ & $49.8 \pm 5.7^{*}$ & $43.6 \pm 4.6^{*}$ \\
\hline Sample size (n) & & 10 & 21 & 21 \\
\hline
\end{tabular}

predictive, and negative predictive values. ${ }^{12}$ Finally, relative proteolytic balance was expressed as the ratio of MMP abundance to a composite TIMP score composed of the sum of TIMP-1, TIMP-2, TIMP-3, and TIMP-4 abundance in each sample. Changes in the ratio of MMP abundance to a composite TIMP score were determined by using a 1-sample mean comparison test with the hypothesized mean for the referent controls set at $100 \%$. All statistical calculations were made using the Stata software package version 8.2 (Stata Corp, College Station, Tex).

\section{RESULTS}

Tissue and plasma measurements of MMPs, TIMPs, and miRs standardized to normal aorta or normal plasma are shown in Figure 1. Absolute value measurements are summarized in Table 1 (Tissue) and Table 2 (Plasma). There were significant differences in the tissue and/or plasma levels of several analytes with respect to control values. Moreover, a differential expression of certain analytes was observed in TAAs from the BAV and TAV groups. For example, tissue levels of miR-1 and miR-21 were differentially altered the TAV group compared with the BAV group (Table 1) Lastly, relative proteolytic balance in the tissue specimens was expressed as the ratio of MMP abundance to a composite TIMP score composed of the sum of TIMP-1, TIMP-2, TIMP-3, and TIMP-4 abundance in each sample, as shown in Appendix Figure 1. Relative to normal aorta, BAV proteolytic balance was significantly increased for MMP-1, MMP-2, and MMP-7, and for decreased MMP-8 and MMP-9. In contrast, TAV proteolytic balance relative to normal aorta was significantly increased only for MMP-1 and decreased for MMP-8 and MMP-9.

Linear regression analysis, performed to identify significant relationships between tissue and plasma levels of each analyte, revealed significant linear relationships only for MMP-8 and TIMP-1, TIMP-3, and TIMP-4. These results are summarized in Figure 2.

Receiver operator characteristic curve analysis was performed to determine if plasma levels of the analytes could serve as biomarker(s) for the presence/absence of TAA disease. The AUC values from the univariate analysis are summarized in Table 3. Following this, forward stepwise multivariable receiver operating characteristics analysis was performed, which revealed specific cassettes of analytes predictive of TAA disease, as depicted in Figure 3. For TAA disease overall, the combination of miR-143, MMP8, and miR-133a maximized AUC values to 0.9660. For TAAs associated with BAV, the combination of MMP-2, TIMP-2, miR-143, miR-133a, and miR-145 maximized AUC values to 0.9766 . For TAAs associated with TAV, the combination of MMP-2, miR-143, and MMP-8 maximized AUC values to 0.9591 .

Logistic regression coefficients and intercepts for biomarkers that were found to be significant predictors of 
TABLE 2. Absolute values for microRNA (miR) expression and protein abundance of matrix metalloproteinases (MMPs) and tissue inhibitors of metalloproteinase (TIMPs) in plasma from patients with healthy aortae and patients with thoracic aortic aneurysm (TAA) with bicuspid aortic valve (BAV) or tricuspid aortic valve (TAV)

\begin{tabular}{|c|c|c|c|c|}
\hline & Plasma analyte & Control & BAV & TAV \\
\hline \multicolumn{5}{|l|}{$\operatorname{miR}\left({ }^{2-\Delta \mathrm{Ct}}\right)$} \\
\hline & $\operatorname{miR}-1\left(1 \times 10^{-3}\right)$ & $70.1 \pm 15.7$ & $70.8 \pm 8.2$ & $72.7 \pm 13.0$ \\
\hline & miR-21 & $42.5 \pm 11.7$ & $36.7 \pm 5.8$ & $32.4 \pm 5.6$ \\
\hline & miR-29a & $7.2 \pm 1.4$ & $6.1 \pm 0.7$ & $5.7 \pm 0.8$ \\
\hline & miR-133a $\left(1 \times 10^{-3}\right)$ & $45.0 \pm 12.7$ & $114.5 \pm 22.9$ & $128.0 \pm 30.0$ \\
\hline & $\operatorname{miR}-143\left(1 \times 10^{-1}\right)$ & $14.4 \pm 2.5$ & $12.2 \pm 1.4$ & $11.3 \pm 1.4$ \\
\hline & $\operatorname{miR}-145\left(1 \times 10^{-2}\right)$ & $94.3 \pm 11.4$ & $69.8 \pm 5.0$ & $84.3 \pm 11.0$ \\
\hline \multicolumn{5}{|l|}{ MMPs (pg/mL) } \\
\hline & MMP-1 & ND & ND & ND \\
\hline & MMP-2 $\left(1 \times 10^{4}\right)$ & $53.4 \pm 4.7$ & $45.6 \pm 3.6$ & $41.9 \pm 3.1$ \\
\hline & MMP-3 $\left(1 \times 10^{3}\right)$ & $22.8 \pm 3.6$ & $16.1 \pm 2.9$ & $19.7 \pm 4.6$ \\
\hline & MMP-7 $\left(1 \times 10^{2}\right)$ & $20.8 \pm 18.4$ & $16.0 \pm 6.3$ & $35.5 \pm 14.5$ \\
\hline & MMP-8 $\left(1 \times 10^{2}\right)$ & $91.0 \pm 28.9$ & $13.3 \pm 3.5^{*}$ & $10.8 \pm 1.3^{*}$ \\
\hline & MMP-9 $\left(1 \times 10^{4}\right)$ & $83.6 \pm 21.5$ & $25.6 \pm 3.5^{*}$ & $41.8 \pm 5.3^{*}, \dagger$ \\
\hline & MMP-12 & ND & ND & ND \\
\hline & MMP-13 $\left(1 \times 10^{2}\right)$ & $24.9 \pm 15.0$ & $11.0 \pm 3.6$ & $8.5 \pm 3.6$ \\
\hline \multicolumn{5}{|l|}{ TIMPs (pg/mL) } \\
\hline & TIMP-1 $\left(1 \times 10^{3}\right)$ & $116.4 \pm 24.7$ & $56.0 \pm 3.2^{*}$ & $63.8 \pm 7.2^{*}$ \\
\hline & TIMP-2 $\left(1 \times 10^{3}\right)$ & $55.2 \pm 2.8$ & $43.6 \pm 1.7^{*}$ & $46.9 \pm 2.4$ \\
\hline & TIMP-3 $\left(1 \times 10^{2}\right)$ & $34.2 \pm 4.6$ & $24.1 \pm 2.8$ & $36.2 \pm 4.6$ \\
\hline & TIMP-4 $\left(1 \times 10^{2}\right)$ & $19.4 \pm 2.9$ & $14.7 \pm 1.2$ & $13.3 \pm 1.1$ \\
\hline Sample size (n) & & 10 & 21 & 21 \\
\hline
\end{tabular}

$N D$, Not detectable. $* P<.05$ versus control. $\dagger P<.05$ versus BAV.

aneurysm development in multivariate analysis were computed.

For all TAAs, the equation: Prob(control/aneur$\mathrm{ysm})=(2.771357 \times \mathrm{miR}-143)-(0.0008061 \times$ MMP-8 $)$ $+(50.81172 \times$ miR-133a $)-3.615998\left(r^{2}, 0.59 ; P<.001\right)$ yielded a positive predictive value of 0.92 , a negative predictive value of 0.58 , a sensitivity of 0.87 , and a specificity of 0.70 .

For the BAV group, the equation: Prob(control/aneur$\mathrm{ysm})=(-0.0000152 \times$ MMP-2 $)-(0.0003459 \times$ TIMP$2)+(25.91564 \times$ miR-133a $)-(8.569773 \times$ miR-145 $)+$ $29.68998\left(r^{2}, 0.73 ; P<.001\right)$ resulted a positive predictive value of 0.95 , a negative predictive value of 1.00 , a sensitivity of 0.95 , and a specificity of 1.00 .

For the TAV group, the equation: Prob(control/aneur$\mathrm{ysm})=(-0.0000284 \times$ MMP-2 $)+(4.211116 \times \mathrm{miR}-143)$ $-(0.0020155 \times$ MMP-8 $)+14.55217\left(r^{2}, 0.67 ; P<.001\right)$ yielded a positive predictive value of 0.89 , a negative predictive value of 0.80 , a sensitivity of 0.89 , and a specificity of 0.80 .

\section{DISCUSSION}

Our knowledge of the pathobiology of TAAs continues to expand and as such, it is becoming more apparent that this information may be used to improve the way we diagnose, track, and treat these serious conditions. Of particular significance is that TAAs of different etiologic subtypes display different biologic patterns, which may allow for personalized health care strategies. Currently, TAAs are diagnosed serendipitously during routine physical examinations or assessments for other disease conditions. A screening test for TAAs would be very valuable to identify those pateints who have asymptomatic but potentially lifethreatening aneurysms, necessitating knowledge of plasma biomarker predictors. As such, this study undertook the task of identifying plasma signatures that could be indicative of specific subtypes of ascending aortic aneurysm disease. This study demonstrated that it was possible to measure a variety of different analytes directly relevant to TAA disease in plasma. Second, very little concordance between plasma measurements and aortic tissue measurements of MMPs, TIMPs, and a specific cassette of miRs was observed. Third, it was shown that aneurysms associated with either BAVs or TAVs displayed different cassettes of tissue and plasma analytes. Finally, it was demonstrated that it was possible to predict with a high degree of specificity and sensitivity the presence of either aneurysm disease in general or specific etiologic subtypes of aneurysm disease in particular using a plasma multianalyte regression strategy. All of the above carries hope that such technology could eventually be configured to a simple plasma measurement that could aid with the screening of patients and perhaps to use these signatures to predict aneurysm activity or changes in aneurysm size. 

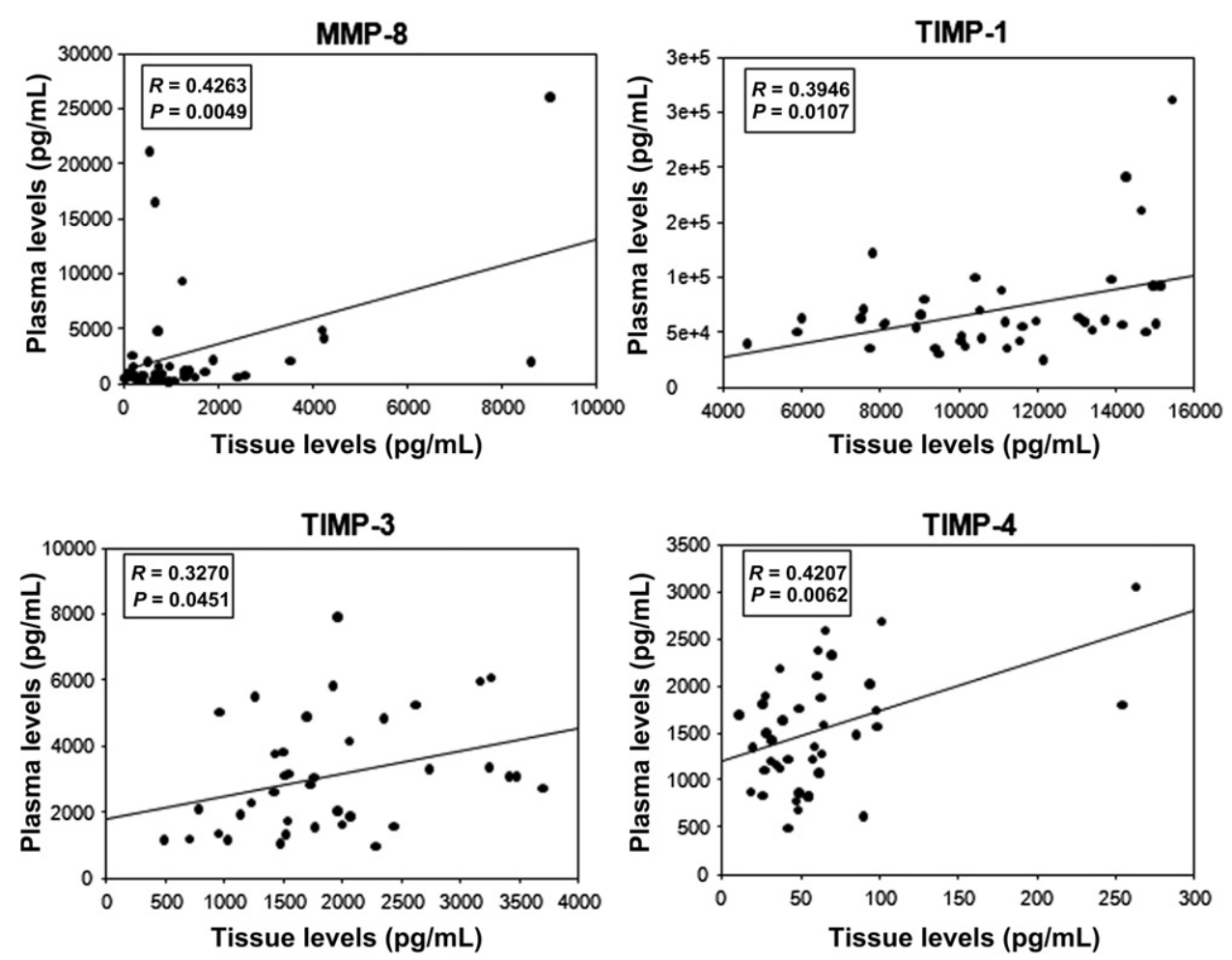

FIGURE 2. Linear regression analysis identifying significant relationships between analyte tissue and plasma levels. Significant relationships were found for matrix metalloproteinase $(M M P)$ 8, tissue inhibitor of metalloproteinase (TIMP) 1, TIMP-3, and TIMP-4.

In our study, a significant number of MMPs, TIMPs, and miRs were measured in aortic tissue. The results were consistent to some extent with findings that $\mathrm{we}^{3,4}$ and others ${ }^{1,2}$

TABLE 3. Area-under-the-curve (AUC) for individual plasma analytes

\begin{tabular}{llll}
\hline & All aneurysms & \multicolumn{1}{c}{ BAV } & \multicolumn{1}{c}{ TAV } \\
\hline miR-1 & $0.4571(.84)$ & $0.4795(.97)$ & $0.4306(.75)$ \\
miR-21 & $0.4538(.46)$ & $0.4850(.65)$ & $0.4211(.37)$ \\
miR-29a & $0.4077(.31)$ & $0.4300(.41)$ & $0.3842(0.35)$ \\
miR-133a & $0.7654(<.01)^{*}$ & $0.7602(<.01)^{*}$ & $0.7712(<.01)^{*}$ \\
miR-143 & $0.3596(.23)$ & $0.3567(.36)$ & $0.3626(.21)$ \\
miR-145 & $0.3385(.21)$ & $0.2700(.02)^{*}$ & $0.4105(.65)$ \\
MMP-1 & & ND & \\
MMP-2 & $0.2615(.03)^{*}$ & $0.3050(.07)$ & $0.2158(.02)^{*}$ \\
MMP-3 & $0.3231(.45)$ & $0.3350(.28)$ & $0.3105(.62)$ \\
MMP-7 & $0.6071(.80)$ & $0.5769(.66)$ & $0.6333(.56)$ \\
MMP-8 & $0.1321(<.01)^{*}$ & $0.1275(<.01)^{*}$ & $0.1368(<.01)^{*}$ \\
MMP-9 & $0.1667(<.01)^{*}$ & $0.0950(<.01)^{*}$ & $0.2421(<.01)^{*}$ \\
MMP-12 & & \multicolumn{1}{c}{$\mathrm{ND}$} & \\
MMP-13 & $0.3590(.08)$ & $0.3889(.20)$ & $0.3333(.10)$ \\
TIMP-1 & $0.2231(<.01)^{*}$ & $0.1850(<.01)^{*}$ & $0.2632(<.01)^{*}$ \\
TIMP-2 & $0.2051(<.01)^{*}$ & $0.1300(<.01)^{*}$ & $0.2842(.03)^{*}$ \\
TIMP-3 & $0.3833(.44)$ & $0.2575(.04)^{*}$ & $0.5158(.75)$ \\
TIMP-4 & $0.3205(.04)^{*}$ & $0.3650(.18)$ & $0.2737(.01)^{*}$ \\
\hline
\end{tabular}

Values are presented as AUC ( $P$ value) for each individual analyte. $B A V$, Bicuspid aortic valve; $T A V$, tricuspid aortic valve; $m i R$, microRNA; $M M P$, matrix metalloproteinase; $N D$, not detectable; TIMP, tissue inhibitor of metalloproteinase. *AUC value is statistically significant in univariate analysis. have made before with regard to differential expression of these analytes for aneurysms of different etiologies. This data further supports the concept that different etiologic subtypes of TAAs display measurable biologic differences that can be used to distinguish meaningfully between these disease processes.

We showed that a broad range of analytes could be measured in the plasma and that it was possible to generate a different cassette of specific analyte profiles for TAAs associated with BAV or TAV. What was interesting with this study was that in general it was not possible to demonstrate a specific correlation between most tissue and plasma analyte levels. The reasons for this are multifactorial and include the fact that many of the biomarkers measured are primarily interstitial molecules and hence it may be difficult to predict how much measurable spillage into plasma would be observed or expected. Furthermore, the half-life of the analytes is variable, making it difficult to correlate plasma and tissue concentrations. Also, tissue and plasma storage and handling affects the ability to accurately measure analytes. Although handling of tissue and plasma is an important and rigorous procedure in the laboratory, it is possible that aberrancies in the storage and processing may have affected the results and decreased the degree of concordance between tissue and plasma levels.

Previous work has underscored the potential for using plasma analytes to predict aortic disease and the results of 

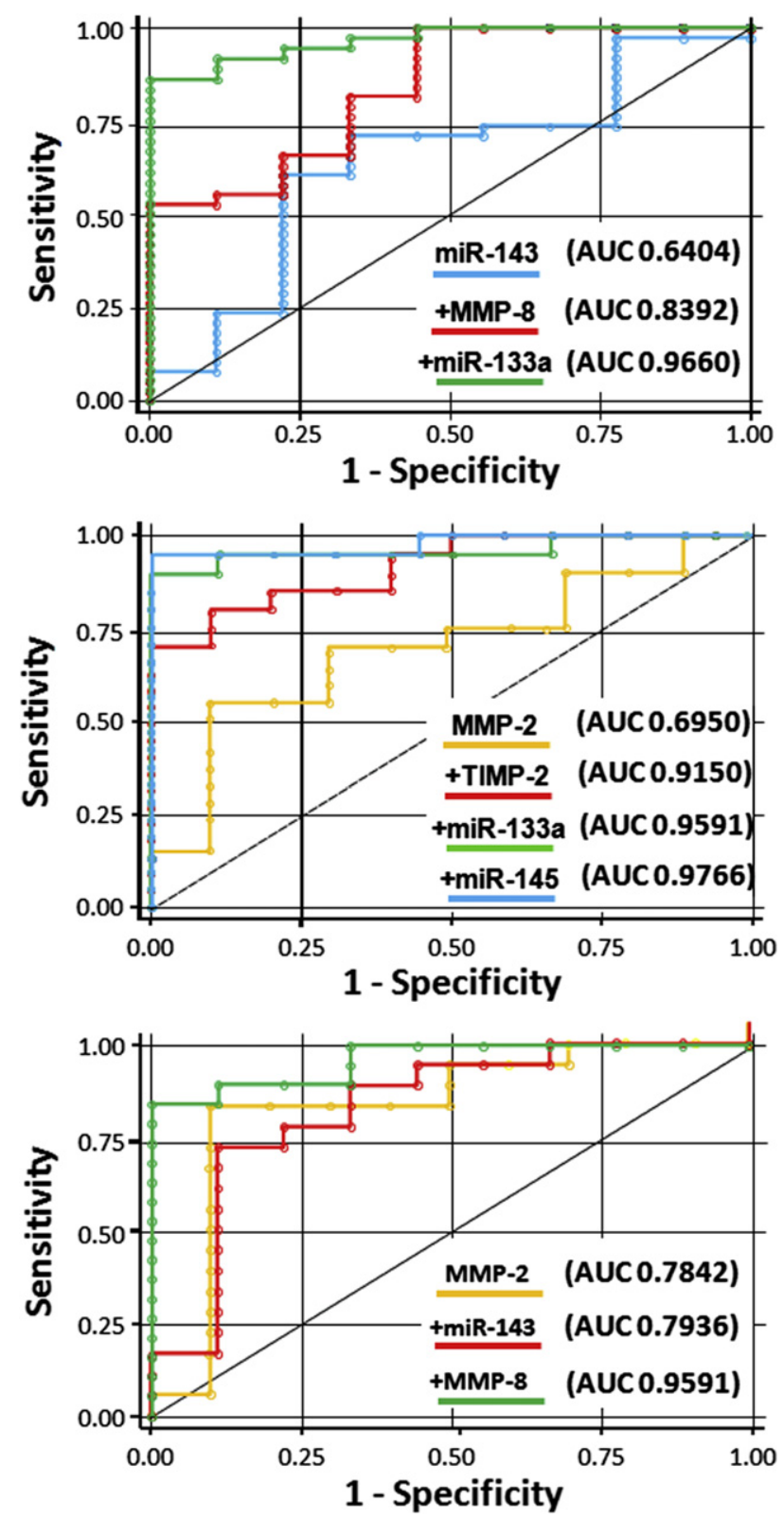

FIGURE 3. Receiver operating characteristic curves to assess plasma aneurysm predictability. Inclusion of plasma analytes using forward stepwise variable selection resulted in different combinations for thoracic aortic aneurism (TAA) in general (top), bicuspid aortic valve-associated TAAs (middle), and tricuspid aortic valve-associated TAAs (bottom) providing high area-under-the curve $(A U C)$ values, indicating high sensitivity and specificity. $M M P$, Matrix metalloproteinase; TIMP, tissue inhibitor of metalloproteinase; $m i R$, microRNA.

interventional therapy. Numerous molecules, including MMPs, C-reactive protein, and D-dimer, have shown promise in the prediction of acute aortic dissection. ${ }^{13} \mathrm{~A}$ recent meta-analysis implicated measurement of circulating MMP-9 as an indicator of abdominal aortic aneurysm disease, ${ }^{14}$ and plasma measurement of MMP-1 and MMP-9 has been shown to be predictive of aortic rupture. ${ }^{15}$ Further, composite measurement of high-density lipoprotein, $\mathrm{C}$-reactive protein, and immunoglobulin $\mathrm{G}$ correlates to abdominal aortic aneurysm size. ${ }^{16}$ In the thoracic aorta, measurement of circulating MMP-9 has been shown to correlate to aortic root dilation. ${ }^{17}$ Interestingly, elevated plasma MMP-9 levels have been used to predict endoleaks after endovascular therapy of the abdominal ${ }^{18}$ and thoracic $^{19}$ aorta. Our work supports the above results and establishes preliminary algorithms for the screening of patients for ascending aortic aneurysm disease.

An important finding in our study is that measurement of single analytes is not predictive of aneurysm disease to any significant level, but a stepwise combination of multiple analytes produces an algorithm that is highly sensitive and specific. As discussed above, a similar trend has been observed for predictions of aortic dissection and abdominal aortic aneurysm. ${ }^{13,16}$ Hence, aneurysms of different etiologies could have specific plasma regression equations containing a composite of analytes that would accurately predict the presence of disease as a screening tool in patients before referral for confirmatory imaging.

It must be understood that this study has significant limitations. The sample size of measurement is relatively small and also the panel of MMPs, TIMPs, and miRs tested, although significant in size, is not comprehensive. Further studies must be undertaken testing a larger bank of analytes to assess for any further significance. In addition, it must be realized that the regression equations generated for different aneurysm subtypes must be tested in a larger bioset for validation. Related to this, postresection plasma samples would be important for validation of the biomarker algorithm. As already discussed, the results of the study may be limited by issues related to obtaining, storing, and processing of tissue and plasma within a multicenter biobank such as the aortic biobank housed at the Medical University of South Carolina. Further refinement of storage protocols and processing should decrease the variability associated with measurements. Because the aortic tissue was snap-frozen and analyzed following homogenization, differentiation of biomarker elaboration between cell types was not possible. This latter point is a challenging area, because in addition to release of biomarkers from cells that have undergone apoptosis, some analytes such as miRs are actively and selectively exported by living cells, ${ }^{20}$ thus significantly confounding the cellular origin issue. Lastly, this observational study does not offer any significant insight regarding which tissue and/or circulating biomarker is a predictor of aortic growth and/or rupture and/or dissection.

The above limitations notwithstanding, the results of this study indicate that specific plasma biosignatures can be generated for aneurysms of different subtypes. These data hold significant importance with regard to the potential 
advancement of diagnosis, tracking, and treatment of thoracic aortic aneurysm disease.

\section{References}

1. Fedak PW, de Sa MP, Verma S, Nili N, Kazemian P, Butany J, et al. Vascular matrix remodeling in patients with bicuspid aortic valve malformations: implications for aortic dilatation. J Thorac Cardiovasc Surg. 2003; 126:797-806.

2. LeMaire SA, Wang X, Wilks JA, Carter SA, Wen S, Won T, et al. Matrix metalloproteinases in ascending aortic aneurysms: bicuspid versus trileaflet aortic valves. J Surg Res. 2005;123:40-8.

3. Ikonomidis JS, Jones JA, Barbour JR, Stroud RE, Clark LL, Kaplan BS, et al. Expression of matrix metalloproteinases and endogenous inhibitors within ascending aortic aneurysms of patients with Marfan syndrome. Circulation. 2006;114: I365-70.

4. Ikonomidis JS, Jones JA, Barbour JR, Stroud RE, Clark LL, Kaplan BS, et al. Expression of matrix metalloproteinases and endogenous inhibitors within ascending aortic aneurysms of patients with bicuspid or tricuspid aortic valves. J Thorac Cardiovasc Surg. 2007;133:1028-36.

5. Jones JA, Stroud RE, O'Quinn EC, Black LE, Barth JL, Elefteriades JA, et al. Selective microRNA suppression in human thoracic aneurysms: relationship of miR-29a to aortic size and proteolytic induction. Circ Cardiovasc Genet. 2011;4:605-13.

6. Small EM, Frost RJ, Olson EN. MicroRNAs add a new dimension to cardiovascular disease. Circulation. 2010;121:1022-32.

7. Elia L, Quintavalle M, Zhang J, Contu R, Cossu L, Latronico MV, et al. The knockout of miR-143 and -145 alters smooth muscle cell maintenance and vascular homeostasis in mice: correlates with human disease. Cell Death Differ. 2009; 16:1590-8.

8. Mestdagh P, Van Vlierberghe P, De Weer A, Muth D, Westermann F, Speleman F, et al. A novel and universal method for microRNA RT-qPCR data normalization. Genome Biol. 2009;10:R64.
9. Meyer SU, Pfaffl MW, Ulbrich SE. Normalization strategies for microRNA profiling experiments: a 'normal' way to a hidden layer of complexity? Biotechnol Lett. 2010;32:1777-88.

10. Ikonomidis JS, Ruddy JM, Benton SM Jr, Arroyo J, Brinsa TA, Stroud RE, et al. Aortic dilatation with bicuspid aortic valves: cusp fusion correlates to matrix metalloproteinases and inhibitors. Ann Thorac Surg. 2012;93:457-63.

11. Essa EM, Zile MR, Stroud RE, Rice A, Gumina RJ, Leir CV, et al. Changes in plasma profiles of matrix metalloproteinases (MMPs) and tissue inhibitors of MMPs in stress-induced cardiomyopathy. J Card Fail. 2012;18:487-92.

12. Altman DG, Bland JM. Diagnostic tests 2: Predictive values. BMJ. 1994;309:102.

13. Wen D, Zhoux L, Li JJ, Hui RT. Biomarkers in aortic dissection. Clin Chim Acta 2011;412:688-95.

14. Takagi H, Manabe H, Kawai N, Goto SN, Umemoto T. Circulating matrix metalloproteinase- 9 concentrations and abdominal aortic aneurysm presence: a meta-analysis. Interact Cardiovasc Thorac Surg. 2009;9:437-40.

15. Wilson WR, Anderton M, Choke EC, Dawson J, Loftus IM, Thompson MM. Elevated plasma MMP1 and MMP9 are associated with abdominal aortic aneurysm rupture. Eur J Vasc Endovasc Surg. 2008;35:580-4.

16. Hellenthal FA, Pulinx B, Welten RJ, Teijink JA, van Dieijen-Visser MP, Wodzig WK, et al. Circulating biomarkers and abdominal aortic aneurysm size. J Surg Res. 2012;176:672-8.

17. Karakaya O, Barutcu I, Esen AM, Dogan S, Saglam M, Karapinar H, et al Relationship between circulating plasma matrix metalloproteinase-9 (gelatinase-B) concentration and aortic root dilatation. Am J Hypertens. 2006;19:361-5.

18. Hellenthal FA, Ten Bosch JA, Pulinx B, Wodzig WK, de Haan MW, Prins MH, et al Plasma levels of matrix metalloproteinase-9: a possible diagnostic marker of successful endovascular aneurysm repair. Eur J Vasc Endovasc Surg. 2012;43:171-2.

19. Monaco M, Stassano P, Di Tommaso L, Iannelli G. Response of plasma matrix metalloproteinases and tissue inhibitor of metalloproteinases to stent-graft surgery for descending thoracic aortic aneurysms. J Thorac Cardiovasc Surg. 2007; 134:925-31.

20. Wang K, Zhang S, Weber J, Baxter D, Galas DJ. Export of microRNAs and microRNA-protective protein by mammalian cells. Nucleic Acids Res. 2010; 38:7248-59.

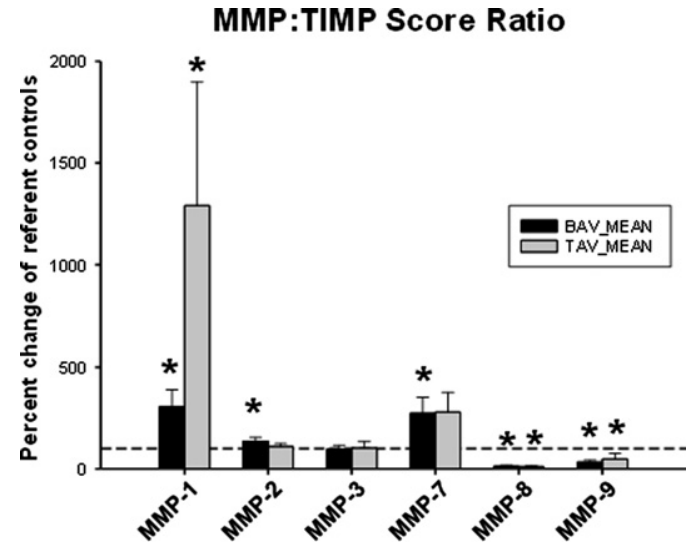

APPENDIX FIGURE 1. Relative proteolytic balance expressed as the ratio of matrix metalloproteinase $(M M P)$ abundance to a composite tissue inhibitor of metalloproteinase (TIMP) score composed of the sum of TIMP-1, TIMP-2, TIMP-3, and TIMP-4. Different profiles of proteolytic balance were observed for the bicuspid aortic valve $(B A V)$ and tricuspid aortic valve $(T A V)$ groups. $* P<.05$ from normal aorta. 